\title{
Estudo de categorias para sistematização de conceitos em gestão do conhecimento
}

\author{
Study of categories for systematization of concepts in knowledge management \\ Narjara Bárbara Xavier Silva ${ }^{1}$, Luana Farias Sales ${ }^{2}$, Jhonathan Divino Ferreira dos Santos ${ }^{3}$ \\ ${ }^{1}$ Universidade Federal do Rio de Janeiro: Rio de Janeiro, Rio de Janeiro, Brasil. ORCID: http://orcid.org/0000-0002-2646-3797 \\ 2 Ministério da Ciência, Tecnologia, Inovações e Comuniações (MCTIC). Distrito Federal, Brasília, Brasil. ORCID: \\ https://orcid.org/0000-0002-3614-2356 \\ ${ }^{3}$ Instituto de Pesquisa Econômica Aplicada (IPEA), Distrito Federal, Brasília, Brasil. ORCID: https://orcid.org/0000-0001-9004-0476
}

Autor para correspondência/Mail to: Narjara Bárbara Xavier Silva, narjara.barbara@gmail.com

Recebido/Submitted: 05 de junho de 2020; Aceito/Approved: 05 de julho de 2020

Copyright (c) 2020 Silva \& Sales \& Santos. Todo o conteúdo da Revista (incluindo-se instruções, política editorial e modelos) está sob uma licença Creative Commons Atribuição-NãoComercial-Compartilhalgual 3.0 Não Adaptada. Ao serem publicados por esta Revista, os artigos são de livre uso em ambientes educacionais, de pesquisa e não comerciais, com atribuição de autoria obrigatória. Mais informações em http://revistas.ufpr.br/atoz/about/submissions\#tcopyrightNotice.

\begin{abstract}
Resumo
Introdução: Com base nos princípios de categorização, o estudo relata uma pesquisa em andamento sobre o categorias para sistematização de conceitos em gestão do conhecimento. Objetivo: Objetiva criar uma taxonomia-de-base-em-conceito para o domínio da Gestão do Conhecimento (GC), cujo resultado poderá minimizar a confusão conceitual entre especialistas, promovendo uma melhor comunicação e também o crescimento da especialidade. Metodologia: Como recurso metodológico da abordagem de análise de domínio, uma revisão sistemática de literatura se fez necessária com o objetivo de identificar e definir as categorias que irão compor o referido esquema de classificação. Conclusão: Como resultado, duas grandes categorias foram criadas, além de sete subcategorias correspondentes. Como etapas seguintes, pretende-se definir os conceitos, que passarão por um processo de sistematização em cadeias hierárquicas, cumprindo assim o objetivo geral da pesquisa que é a criação de uma taxonomia para o domínio da GC.
\end{abstract}

Palavras-chave: Gestão do Conhecimento; Estudo de Categorias; Revisão Sistemática de Literatura; Teorias da Organização do Conhecimento; Taxonomia.

\begin{abstract}
Introduction: Based on the categorization principles, the study reports on-going research about the categories to systematize the concepts in knowledge management. Objective: It aims to create a concept-based Taxonomy for the Knowledge Management (KM) domain, the outcome of which may minimize conceptual misunderstanding among experts, promoting better communication and also the growth of the specialty. Method: As a methodological resource of the domain analysis approach, a systematic literature review was necessary in order to identify and define the categories that will compose the classification scheme. Conclusion: As a result, two major categories were created, plus seven corresponding subcategories. The following steps are intended to define the concepts, which will go through a process of systematization in hierarchical chains, thus fulfilling the general objective of the research that is the creation of a taxonomy for the domain of KM.
\end{abstract}

Keywords: Knowledge Management; Study of Categories; Systematic Review of Literature; Theories of Knowledge Organization; Taxonomy.

\section{INTRODUÇÃO}

Dada a multidisciplinaridade da Gestão do Conhecimento (GC) desde sua origem, é possível encontrar diferentes abordagens sobre esta temática na literatura acadêmica com crescente produção científica nas últimas décadas, o que acarreta diversidade terminológica. Essa diversidade impacta diretamente na distribuição dos termos entre suas categorias no momento de sistematizar os conceitos relacionados ao domínio da GC, encontrando na taxonomia um modelo metodológico de organização desses conceitos. Assim, o problema que se apresenta é que, nesta especialidade, cujo padrão terminológico é um instrumento de grande importância para a condução de qualquer trabalho (Terra, Schouerl, Vogel, \& Franco, 2009), ainda há carência de um instrumento que padronize e sistematize seus próprios conceitos.

Por outro lado, trabalhar os conceitos que envolvem a GC é um desafio, pois cada subárea que a compõe compreende-os de uma forma. Nesse sentido, faz-se necessário um estudo aprofundado dos princípios de categorização (Dahlberg, 1981; Ranganathan, 1937), a fim de auxiliar na compreensão dos conceitos que fazem parte do domínio da Gestão do Conhecimento. Categorização é entendida como um processo dedutivo de "analisar o domínio a partir de recortes conceituais que permitem determinar a identidade dos conceitos (categorias) que fazem parte desse domínio" (Campos \& Gomes, 2008).

Categorias, por sua vez, são definidas por Campos, Gomes, e Oliveira (2013) como elementos essenciais para a elaboração de toda e qualquer taxonomia, pois possibilita a visão de uma área ou assunto como um sistema, permitindo identificar nele os aspectos pelos quais tal área/assunto pode ser abordado. Nessa concepção, o presente trabalho é parte de uma pesquisa em andamento que visa propor uma Taxonomia-de-base-em-conceito 
para a Gestão do Conhecimento na área da Ciência da Informação (CI), a partir da delimitação e definição de categorias que, a priori, propiciará lógica para o referido esquema classificatório.

Assim, nesse primeiro momento, pretende-se analisar, categorizar e definir as diferentes abordagens que compõem o domínio da GC, tomando como base os principais autores referenciados na área da CI e suas respectivas obras originais. Para isso, uma revisão sistemática de literatura se fez necessária, definida por Sampaio e Mancini (2007) e Galvão e Pereira (2014) como uma investigação de caráter retrospectivo focada em uma questão precisa, cujo objetivo é identificar, selecionar, analisar e sintetizar as evidências relevantes disponíveis em estudos primários sobre um determinado tema, a partir do uso de métodos sistemáticos e explícitos.

Como resultado desta pesquisa, pretende-se contribuir para a sistematização de conceitos em Gestão do Conhecimento no âmbito da Ciência da Informação servindo-se de suporte para a teoria e prática na especialidade da GC.

\section{MÉTODO}

A Análise de Domínio (AD) tem encontrado amplo espaço na Organização do Conhecimento, usada para fins epistemológicos e teóricos, cujos conceitos são aprofundados, gerando arcabouço teórico para aquele estudo. Por outro lado, como método, a AD não se restringe a ela, podendo ser usada para auxiliar no desenvolvimento de instrumentos de classificação, cujos conceitos são aplicados metodologicamente na área do conhecimento da pesquisa (Gheno, 2017). Neste caso, na Gestão do Conhecimento.

Segundo Smiraglia (2011 apud Guimarães, 2014, p. 15), a Análise de Domínio é definida como um "estudo dos aspectos teóricos de dado entorno, geralmente representado por uma literatura ou comunidade de pesquisadores, constituindo um meio para a geração de novo conhecimento acerca da interação de dada comunidade científica com a informação". De outro modo, o domínio, objeto de análise, é mapeado por Guimarães (2014, p. 17) como sendo:

um conjunto de comunidades de pensamento ou comunidades discursivas que integram a divisão social do trabalho (Hjorland; Albrechtsen, 1995, p. 401 apud Guimarães, 2014, p. 17); uma área de especialidade, um conjunto literário ou um grupo de pessoas trabalhando juntas numa organização (Mai, 2005, p. 605 apud Guimarães, 2014, p. 17), ou, ainda, uma área de conhecimento, atividade, interesse ou aplicação com limites definidos (Lloréns et al., 2004 apud Guimarães, 2014, p. 17).

Nesse sentido, conforme Guimarães (2014), o domínio é resultado da aplicação de um princípio inerente à própria organização do conhecimento - a categorização.

Como recurso metodológico da análise de domínio (Hjørland, 2002), uma revisão sistemática de literatura (systematic literature review - SLR, em inglês) se fez necessária, com o objetivo de identificar e definir as categorias que irão compor a Taxonomia-de-base-em-conceito ${ }^{1}$ para o domínio da Gestão do Conhecimento. Iniciadas na década de 1950 como um novo delineamento de pesquisa e consolidadas na área da saúde no fim da década de 1980 (Galvão \& Pereira, 2014), as revisões consideradas como sistemáticas são vistas atualmente como um método aplicável em qualquer área de conhecimento.

Segundo Sampaio e Mancini (2007) e Galvão e Pereira (2014), diferentemente das convencionais revisões narrativas (gerais sobre um determinado tema) ou integrativas (diferentes delineamentos sobre a mesma temática), as revisões sistemáticas se caracterizam como uma investigação de caráter retrospectivo focada em uma questão precisa, cujo objetivo é identificar, selecionar, analisar e sintetizar as evidências relevantes disponíveis em estudos primários sobre um determinado tema, a partir do uso de métodos sistemáticos e explícitos.

A SRL adota quatro fases para o alcance dos seus objetivos, são elas: planejamento, amostragem, análise e relato (Garza-Reyes, 2015). Numa visão mais detalhada, Sampaio e Mancini (2007) mencionam que os métodos para elaboração de revisões sistemáticas preveem: (1) definição da pergunta de pesquisa; (2) busca de evidências na literatura; (3) revisão e seleção dos estudos; (4) análise da qualidade metodológica dos estudos; e (5) apresentação dos resultados.

Com base nos autores supracitados, a Figura 1 ilustra as fases da SLR e os métodos e ferramentas utilizadas neste estudo para suportar seus estágios.

\footnotetext{
${ }^{1} \mathrm{O}$ termo é considerado pelos autores como uma locução substantiva (conjunto de duas ou mais palavras que têm a função de substantivo) e, apesar de o novo Acordo Ortográfico não empregar, em geral, hífen em locuções, optou-se pelo seu uso com a finalidade de dar ênfase ao tipo de taxonomia em questão (de base em conceitos), considerando tal termo uma exceção à regra.
} 


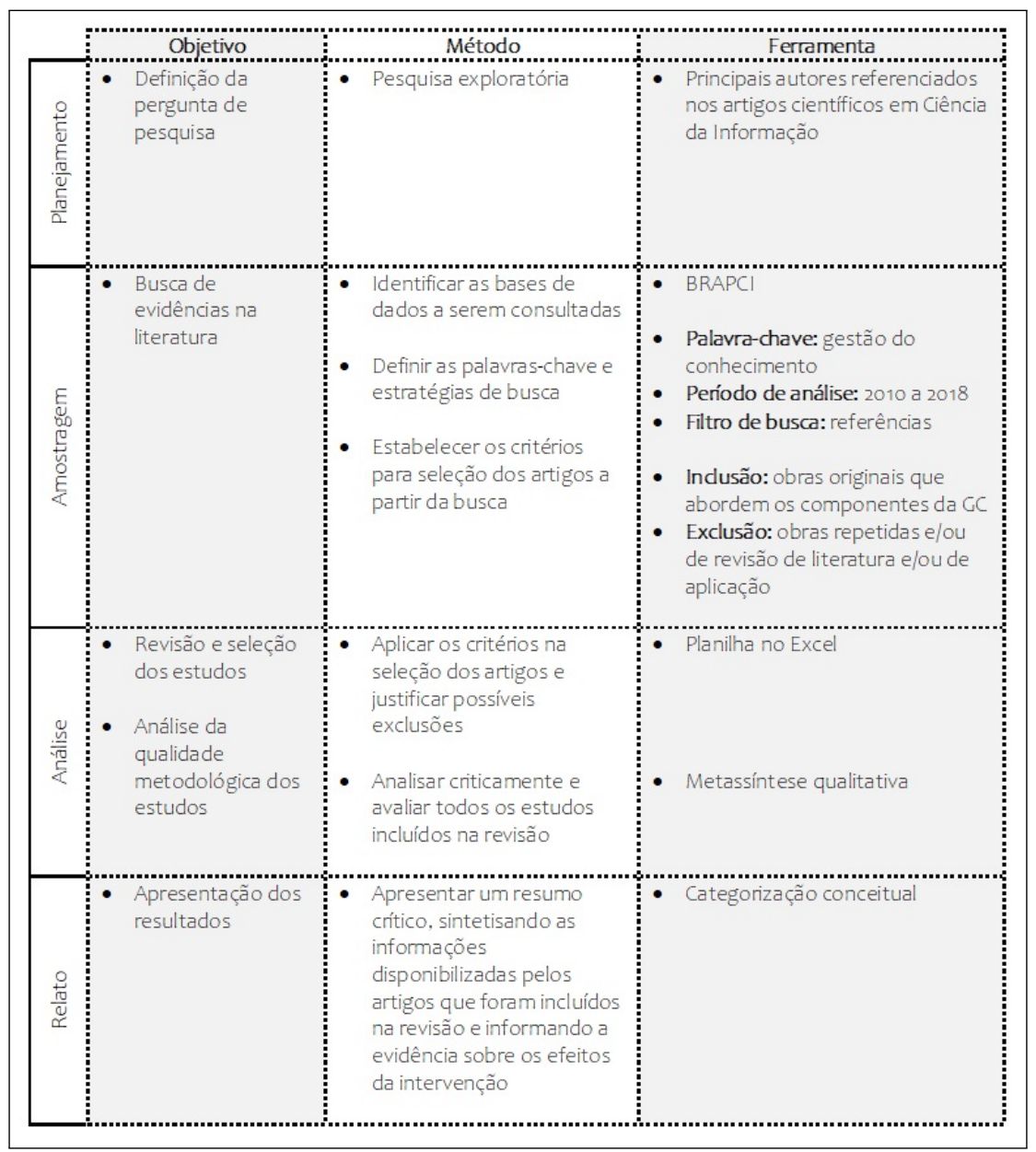

Figura 1. Síntese metodológica de SLR.

Fonte: Elaboração própria, com base em Sampaio e Mancini (2007) e Garza-Reyes (2015)

Na etapa de amostragem, foram utilizadas duas estratégias de busca de evidências na literatura. A primeira delas foi a escolha da Base de Dados Referencial de Artigos de Periódicos em Ciência da Informação (BRAPCI) para consulta, que conta hoje $\mathrm{e}^{2}$ com 19.255 trabalhos em revistas científicas e 2.592 trabalhos em eventos, podendo considerá-la o principal acervo de publicações brasileiras em CI. A segunda estratégia foi utilizar a palavra-chave "gestão do conhecimento" para recuperação de referências contidas nas produções científicas publicadas no período de 01 de janeiro de 2010 a 31 de agosto de $2018^{3}$.

Como critérios para seleção dos artigos, a partir da busca, incluíram-se as obras originais que abordavam os componentes da GC, a exemplo de livros (capítulos ou obras completas) e teses de doutorado, pois normalmente trazem novos conhecimentos para uma determinada área acadêmica. Por outro lado, excluíram-se os demais trabalhos de conclusão de curso de graduação, especialização e mestrado, além de entrevistas, textos de blog, sites e materiais de participação em eventos, disseminadas, muitas vezes, como revisão de literatura e/ou aplicação da GC nos diferentes contextos sociais e organizacionais.

A etapa de análise objetivou revisar e selecionar os estudos de acordo com os critérios de inclusão e exclusão (ver Figura 1), seguida da análise da qualidade metodológica dos estudos selecionados, por meio do método de avaliação denominado metassíntese qualitativa, definida por Zimmer (2006, p. 312 apud Lopes e Fracolli, 2008 , p. 774) como "uma modalidade de estudo qualitativo que utiliza os dados dos achados de outros estudos qualitativos relativos ao mesmo tema ou a temas correlacionados". Tal método pode ser aplicado por meio de relações entre as pesquisas, como comparações, semelhanças e diferenças. Neste estudo, a qualidade metodológica foi analisada a partir da própria abordagem adotada pelos autores, ou seja, identificando se, de fato, a publicação trazia novos conhecimentos para o domínio da GC.

Como parte da quarta e última etapa da SRL, as informações coletadas foram sintetizadas e disponibilizadas a partir da categorização, compreendida como um "processo que requer pensar um domínio de forma dedutiva, ou seja, determinar as classes de maior abrangência dentro da temática escolhida" (Campos \& Gomes, 2008). Ainda

\footnotetext{
${ }^{2}$ Dados coletados em 31 de agosto de 2018.

${ }^{3} \mathrm{O}$ período de análise considerou o intervalo de contínuo crescimento das publicações científicas em Gestão do Conhecimento na Ciência da Informação, tomando como base as produções em um dos seus principais ambientes de comunicação científica da área no Brasil - os Anais do Encontro Nacional de Pesquisa em Ciência da Informação (ENANCIB) (Duarte, Satur, Lira, Silva, \& Lima, 2015)
} 
para as autoras, "aplicar a categorização é analisar o domínio a partir de recortes conceituais que permitem determinar a identidade dos conceitos (categorias) que fazem parte desse domínio" (Campos \& Gomes, 2008).

\section{RESULTADOS}

Após a adoção do termo de busca "gestão do conhecimento" na BRAPCI, utilizando a opção "referências" como filtro de pesquisa, foram recuperadas 657 referências em português. Em seguida foram adotados os critérios de inclusão e exclusão (ver Figura 1), utilizando uma planilha no Excel como suporte ao tratamento dos dados. Ao final, restaram 109 referências.

Para possibilitar o estudo aprofundado da literatura acadêmica referenciada nas publicações científicas da BRAPCI, com a finalidade de identificar a abordagem central adotada por seus respectivos autores, apenas as obras mais referenciadas, com três ou mais menções, foram selecionadas para a pesquisa. Restaram, portanto, 28 obras nacionais para análise, conforme Quadro 1, cujas referências estão ordenadas por ano de publicação. Ressalta-se que, nos casos de obras organizadas por autor(es), foram excluídas as obras completas, independentemente do número de menções, e incluídos os capítulos correspondentes referenciados mais de duas vezes. Assim, evitou-se a confluência de diferentes abordagens numa mesma obra.

OBRA REFERENCIADA

DRUCKER, P. F. O advento da nova organização. In: HARVARD BUSINESS REVIEW.
7

Gestão do Conhecimento. Rio de Janeiro: Campus, 2000.

GARVIN; D. A. Construindo a organização que aprende. In: HARVARD BUSINESS REVIEW. Gestão do Conhecimento. Rio de Janeiro: Campus; 2000.

TERRA, José Cláudio Cyrineu. Gestão do conhecimento: o grande desafio empresarial: uma abordagem baseada no aprendizado e na criatividade. São Paulo: Negócio, 2000.

STOLLENWERK, Maria Fátima Ludovico. Gestão do conhecimento: conceitos e modelos. In: TARAPANOFF, Kira. Inteligência Organizacional e Competitiva. Brasília: Editora Universidade de Brasília, 2001.

BUKOWITZ, W. R.; WILLIAMS, R. L. Manual de gestão do conhecimento: ferramentas e técnicas que criam valor para a empresa. Porto Alegre: Bookman, 2002.

FLEURY, M. T. L.; OLIVEIRA JR, M. M. Aprendizagem e gestão do conhecimento. In: FLEURY, M. T. L. (Org.). As pessoas na organização. São Paulo: Gente, 2002.

PROBST, G.; RAUB, S.; ROMHARDT, K. Gestão do conhecimento: os elementos construtivos do sucesso. Porto Alegre: Bookman, 2002.

CIANCONI, Regina de Barros. Gestão do conhecimento: visão de indivíduos e organizações no Brasil. 2003. 297f. Tese (Doutorado) - Programa de Pós-Graduação em Ciência da Informação, Instituto Brasileiro de Informação em Ciência e Tecnologia (IBICT), Universidade Federal do Rio de Janeiro (UFRJ), Rio de Janeiro, 2003.

BATISTA, F. Governo que aprende: gestão do conhecimento em organizações do executivo federal. Brasília: IPEA, 2004.

GUIMARÃES, J. As políticas de Indexação como elemento para a gestão do conhecimento nas organizações. In: VIDOTTI, S. A. G. (Org.). Tecnologia e conteúdos informacionais: abordagens teóricas e práticas. São Paulo: Polis, 2004.

SANTIAGO JUNIOR, J. R. S. Gestão do conhecimento: a chave para o sucesso empresarial. São Paulo: Novatec, 2004.

ALVARENGA NETO, R. C. D. de. Gestão do conhecimento em organizações: proposta de mapeamento conceitual integrativo. 2005. 400f. Tese (Doutorado) - Programa de Pós-Graduação em Ciência da Informação, Universidade Federal de Minas Gerais (UFMG), Belo Horizonte, 2005.

CARVALHO, R. B. Intranets, portais corporativos e gestão do conhecimento: análise das experiências de organizações brasileiras e portuguesas. 2006. 281f. Tese (Doutorado) - Programa de Pós-Graduação em Ciência da Informação, Universidade Federal de Minas Gerais (UFMG), Belo Horizonte, 2006.

FIALHO, F. A. P.; MACEDO, M.; SANTOS, N. dos; MITIDIERI, T. da C. Gestão do conhecimento e aprendizagem: as estratégias competitivas da sociedade pós-industrial. Florianópolis: Visual Books, 2006.

LIMA-MARQUES, Mamede; MACEDO, Flávia Lacerda Oliveira de. Arquitetura da informação: base para a gestão do conhecimento. In: TARAPANOFF, Kira (Org.). Inteligência, informação e conhecimento. Brasília: IBICT, 2006. 
MCINERNEY, Claire R. Compartilhamento e gestão do conhecimento: profissionais da informação em um ambiente de confiança mútua. In: TARAPANOFF, Kira (Org.). Tradução de Ulf Gregor Baranow. Inteligência, informação e conhecimento. Brasília: IBICT, 2006.

ROSINI, M.; PALMISANO, A. Administração de sistemas de informação e a gestão do conhecimento. São Paulo: Pioneira Thomson Learning, 2006

WILSON, T. D. A problemática da gestão do conhecimento. In: TARAPANOFF, K. (Org.). Inteligência, informação e conhecimento. Brasília: IBICT, Unesco, 2006.

FRESNEDA, P. S. V.; GONÇALVES, S. M. G. A experiência brasileira na formulação de uma proposta de política de gestão do conhecimento para a administração pública federal. Brasília: Câmara dos Deputados, 2007.

INNARELLI, Humberto Celeste. Preservação digital e seus Dez Mandamentos. In: SANTOS, Vanderlei Batista dos; INNARELLI, Humberto Celeste; SOUZA, Renato Tarciso Barbosa de. Arquivística: temas contemporâneos: classificação, preservação, gestão do conhecimento. Brasília: SENAC, 2007.

SANTOS, Vanderlei Batista dos. A prática arquivística em tempos de gestão do conhecimento. In: SANTOS, Vanderlei Batista dos; INNARELLI, Humberto Celeste; SANTOS, Renato Tarciso Barbosa (Org.). Arquivística: temas contemporâneos: classificação, preservação digital, gestão do conhecimento. Brasília, DF: SENAC, 2007.

SOUSA, Renato Tarciso Barbosa de. A classificação como função matricial do que fazer arquivístico. In: SANTOS, Vanderlei Batista dos; INNARELLI, Humberto Celeste; SOUSA, Renato Tarciso Barbosa de (Org.). Arquivística: temas contemporâneos: classificação, preservação digital, gestão do conhecimento. Brasília: SENAC, 2007.

NONAKA, Ikujiro. A empresa criadora do conhecimento. In: NONAKA, Ikujiro; TAKEUCHI, Hirotaka. (Org.). Gestão do conhecimento. Porto Alegre: Bookman, 2008.

NONAKA, Ikujiro; TAKEUCHI, Hirotaka. Teoria da criação do conhecimento organizacional. In: TAKEUCHI, Hirotaka; NONAKA, Ikujiro. Gestão do conhecimento. Porto Alegre: Bookman, 2008.

SILVA, H. M. da; VALENTIM, M. L. P. Modelos de gestão do conhecimento aplicados à ambientes empresariais. In: VALENTIM, M. L. P. (Org.). Gestão da informação e do conhecimento no âmbito da Ciência da Informação. São Paulo: Polis, 2008.

SORDI, J. O. de. Administração da informação: fundamentos e práticas para uma nova gestão do conhecimento. São Paulo: Saraiva, 2008.

TAKEUCHI, H.; NONAKA, I. Criação e dialética do conhecimento. In: Gestão do conhecimento. Porto Alegre: Bookman, 2008.

TERRA, José Claudio Cyrineu; GORDON, Cindy. Portais Corporativos: a revolução na Gestão do Conhecimento. São Paulo: Negócio Editora, 2011.

Quadro 1. Obras nacionais mais referenciadas na BRAPCI

Fonte: Elaboração própria, a partir da coleta de dados na Brapci (2018)

Mediante revisão sistemática das obras referenciadas e aplicação da categorização conceitual, considerando aquelas acessíveis para leitura, o domínio da GC foi classificado em duas grandes categorias, são elas: Campo Científico e Sistema de Gestão. As abordagens que compõem o Campo da GC podem ser categorizadas a partir dos Fundamentos Teóricos e das Abordagens Disciplinares que o compõe. Já o Sistema de GC pode ser classificado em cinco subcategorias, a saber: Programa, Projetos, Processo, Práticas e Produtos.

Para uma melhor compreensão do Quadro 2, destacam-se algumas observações. A primeira é que, apesar de, em sua obra, o autor Batista (2004) identificar Banco de Talentos e Oportunidades (TAO) e Banco de Conhecimentos como práticas de gestão do conhecimento, entende-se que ambos se tratam de produtos, pois os mesmos não remetem à ação, mas geram ou resultam de ações/práticas em GC. A segunda observação é que tais produtos em GC podem ser tecnológicos, especificamente as Tecnologias da Informação e Comunicação (TIC), ou não tecnológicos, quando suportam artefatos tradicionais. 


\begin{tabular}{|c|c|c|c|}
\hline CATEGORIA & SUBCATEGORIA & AUTORES & ABORDAGEM \\
\hline \multirow[t]{2}{*}{ Campo } & $\begin{array}{l}\text { Fundamentos teó- } \\
\text { ricos }\end{array}$ & Wilson (2006) & $\begin{array}{l}\text { Escopo da Gestão do Conhecimento em periódicos } \\
\text { científicos por áreas de conhecimento. }\end{array}$ \\
\hline & $\begin{array}{l}\text { Abordagens disci- } \\
\text { plinares }\end{array}$ & $\begin{array}{l}\text { Alvarenga } \\
\text { Neto (2005) }\end{array}$ & $\begin{array}{l}\text { Mapeamento conceitual da Gestão do Conhecimento: } \\
\text { integração de conceitos de várias disciplinas (Admi- } \\
\text { nistração, Ciência da Informação, Ciência da Compu- } \\
\text { tação), integração de várias atividades (Gestão de Do- } \\
\text { cumentos, Mapeamento de Competências, Mapea- } \\
\text { mento de Processos, Criação de contexto capacitante } \\
\text { ou "ba"), integração de abordagens gerenciais e ferra- } \\
\text { mentas (Gestão da Inovação, Tácito e Explícito, BSC), } \\
\text { integração de diferentes áreas de conhecimento (RH, } \\
\text { Finanças, TI, Marketing, Estratégia). }\end{array}$ \\
\hline \multirow[t]{6}{*}{ Sistema } & \multirow[t]{4}{*}{ Programa } & Terra (2000) & $\begin{array}{l}\text { Sete dimensões da prática gerencial estruturadas em } \\
\text { três níveis interconectados, que são: estratégico (alta } \\
\text { administração), organizacional (políticas de recursos } \\
\text { humanos, cultura organizacional e estrutura organi- } \\
\text { zacional) e infraestrutura (sistemas de informação e } \\
\text { mensuração de resultados). }\end{array}$ \\
\hline & & $\begin{array}{l}\text { Stollenwerk } \\
\text { (2001) }\end{array}$ & $\begin{array}{l}\text { Modelo genérico de gestão do conhecimento - Fato- } \\
\text { res facilitadores: Liderança; Cultura Organizacional; } \\
\text { Mediação e Avaliação; e Tecnologia da Informação. }\end{array}$ \\
\hline & & $\begin{array}{l}\text { Cianconi } \\
(2003)\end{array}$ & $\begin{array}{l}\text { Facetas da Gestão do Conhecimento: gestão da cul- } \\
\text { tura organizacional, gestão de talentos e dos relacio- } \\
\text { namentos internos, gestão de competências e aprendi- } \\
\text { zagem organizacional, gestão dos relacionamentos ex- } \\
\text { ternos, gestão dos processos e das melhores práticas } \\
\text { organizacionais, gestão dos acervos e dos conteúdos } \\
\text { informacionais, gestão da tecnologia e dos sistemas } \\
\text { de informação e mensuração de ativos intangíveis. }\end{array}$ \\
\hline & & $\begin{array}{l}\text { Fresneda; } \\
\text { Gonçalves } \\
\text { (2007) }\end{array}$ & $\begin{array}{l}\text { Premissas básicas e diretrizes para implementação de } \\
\text { uma política de Gestão do Conhecimento integrada e } \\
\text { interorganizacional, que articule os diversos órgãos } \\
\text { da administração direta, criando redes de correspon- } \\
\text { sabilidade em relação aos processos de criação, disse- } \\
\text { minação e compartilhamento de conhecimentos ne- } \\
\text { cessários ao aumento de eficiência dos serviços pres- } \\
\text { tados à sociedade pelas áreas governamentais. Den- } \\
\text { tre as discussões, evidenciam-se algumas questões re- } \\
\text { levantes para a formulação da política de GC: cultura, } \\
\text { sensibilização e capacitação para gestão do conheci- } \\
\text { mento; foco no compartilhamento de conhecimentos; } \\
\text { e tecnologia. }\end{array}$ \\
\hline & Projetos & $\begin{array}{l}\text { Lima- } \\
\text { Marques; } \\
\text { Macedo } \\
(2006)\end{array}$ & $\begin{array}{l}\text { Proposta de modelo de arquitetura da informação que } \\
\text { representa os processos básicos do ciclo da informa- } \\
\text { ção, sobrepostos em três níveis, a saber: metamodela- } \\
\text { gem (análise do contexto do ambiente informacional } \\
\text { como um todo e estabelecimento das diretrizes), mo- } \\
\text { delagem (são definidos os modelos de identificação, } \\
\text { captura, armazenamento, representação, organização } \\
\text { e comunicação dos conteúdos do sistema de informa- } \\
\text { ção) e aplicação (aplicam-se as teorias, modelos, téc- } \\
\text { nicas e tecnologias idealizadas nos níveis anteriores } \\
\text { para a implementação do sistema de informação, com } \\
\text { seus produtos e serviços). }\end{array}$ \\
\hline & Processo & $\begin{array}{l}\text { Stollenwerk } \\
\text { (2001) }\end{array}$ & $\begin{array}{l}\text { Modelo genérico de gestão do conhecimento: - Pro- } \\
\text { cessos: Identificação; Captura; Seleção e Validação; } \\
\text { Organização e Armazenagem; Compartilhamento: } \\
\text { Acesso e Distribuição; Aplicação; e Criação do Co- } \\
\text { nhecimento. }\end{array}$ \\
\hline
\end{tabular}




\begin{tabular}{|c|c|c|c|}
\hline \multirow{11}{*}{ Sistema } & \multirow{5}{*}{ Processo } & $\begin{array}{l}\text { Bukowitz } \\
\text { e Williams } \\
\text { (2002) }\end{array}$ & $\begin{array}{l}\text { Processo de GC classificado em dois cursos de ações } \\
\text { (e suas etapas) integradas simultaneamente à organi- } \\
\text { zação, a saber: curso tático (obtenha, utilize, aprenda } \\
\text { e contribua) e estratégico (avalie, construa / mante- } \\
\text { nha e descarte). }\end{array}$ \\
\hline & & $\begin{array}{l}\text { Probst, Raub } \\
\text { e Romhardt } \\
\text { (2002) }\end{array}$ & $\begin{array}{l}\text { Processos essenciais de gestão do conhecimento, que } \\
\text { são: aqueles relacionados aos fluxos informacionais } \\
\text { (identificação do conhecimento, aquisição do conheci- } \\
\text { mento, desenvolvimento do conhecimento, comparti- } \\
\text { Ihamento e distribuição do conhecimento, utilização } \\
\text { do conhecimento e retenção do conhecimento), além } \\
\text { de dois construtos estratégicos (metas de conheci- } \\
\text { mento e avaliação do conhecimento). }\end{array}$ \\
\hline & & $\begin{array}{l}\text { Mclnerney } \\
\text { (2006) }\end{array}$ & $\begin{array}{l}\text { Compartilhamento do conhecimento e o elemento } \\
\text { "confiança" como fator ambiental básico para sua efe- } \\
\text { tividade. }\end{array}$ \\
\hline & & $\begin{array}{l}\text { Nonaka } \\
(2008)\end{array}$ & $\begin{array}{l}\text { Quatro modos de conversão do conhecimento para } \\
\text { a criação do conhecimento organizacional: Socializa- } \\
\text { ção (tácito » tácito), Combinação (explícito » explícito), } \\
\text { Internalização (explícito » tácito) e Externalização (tá- } \\
\text { cito » explícito). }\end{array}$ \\
\hline & & $\begin{array}{l}\text { Nonaka; Ta- } \\
\text { keuchi (2008) }\end{array}$ & $\begin{array}{l}\text { Processo de criação do conhecimento: Socialização } \\
\text { (compartilhar e criar conhecimento tácito através de } \\
\text { experiência direta), Externalização (articular conheci- } \\
\text { mento tácito através do diálogo e da reflexão), Combi- } \\
\text { nação (sistematizar e aplicar o conhecimento explícito } \\
\text { e a informação) e Internalização (aprender e adquirir } \\
\text { novo conhecimento tácito na prática). }\end{array}$ \\
\hline & \multirow[t]{3}{*}{ Práticas } & Batista (2004) & $\begin{array}{l}\text { Práticas de Gestão do Conhecimento em instituições, } \\
\text { a exemplo: Mapeamento dos conhecimentos organi- } \\
\text { zacionais, Comunidades de Prática (CdP), Mentoring, } \\
\text { Grupos de Revisão de Projetos (Peer Review), Univer- } \\
\text { sidade Corporativa, Alocação de pessoal com base em } \\
\text { competências. }\end{array}$ \\
\hline & & $\begin{array}{l}\text { Innarelli } \\
(2007)\end{array}$ & $\begin{array}{l}\text { Prática da preservação documental a partir dos dez } \\
\text { mandamentos. }\end{array}$ \\
\hline & & $\begin{array}{l}\text { Guimarães } \\
\text { (2004) }\end{array}$ & $\begin{array}{l}\text { Indexação: dimensão de tratamento do conteúdo in- } \\
\text { formacional. }\end{array}$ \\
\hline & \multirow[t]{3}{*}{ Produtos } & Batista (2004) & $\begin{array}{l}\text { Produtos de Gestão do Conhecimento em instituições, } \\
\text { a exemplo: Banco de Talentos e Oportunidades (TAO) } \\
\text { e Banco de Conhecimentos. }\end{array}$ \\
\hline & & $\begin{array}{l}\text { Carvalho } \\
(2006)\end{array}$ & $\begin{array}{l}\text { Tecnologias da Informação e Comunicação (TIC) no } \\
\text { suporte ao processo de Gestão do Conhecimento. Al- } \\
\text { guns exemplos: Socialização (sistemas de mapas de } \\
\text { conhecimento e portais de conhecimento), Externali- } \\
\text { zação (ferramentas de Groupware e Workflow, além } \\
\text { dos sistemas baseados em inteligência artificial e dos } \\
\text { portais de conhecimento), Internalização (ferramen- } \\
\text { tas de suporte à inovação), e Combinação (sistemas de } \\
\text { intranet, Gerenciamento Eletrônico de Documentos } \\
\text { (GED), Business Intelligence, Inteligência Competitiva, } \\
\text { e os portais de conhecimento). }\end{array}$ \\
\hline & & $\begin{array}{l}\text { Terra e Gor- } \\
\text { don (2011) }\end{array}$ & $\begin{array}{l}\text { Portal corporativo como plataforma de suporte ao } \\
\text { processo de gestão do conhecimento, a partir de uma } \\
\text { interface única que integra diferentes tecnologias da } \\
\text { informação e comunicação para a otimização dos flu- } \\
\text { xos informacionais. }\end{array}$ \\
\hline
\end{tabular}

Quadro 2. Análise das obras e abordagem dos respectivos autores Fonte: Dados da Pesquisa (2020) 


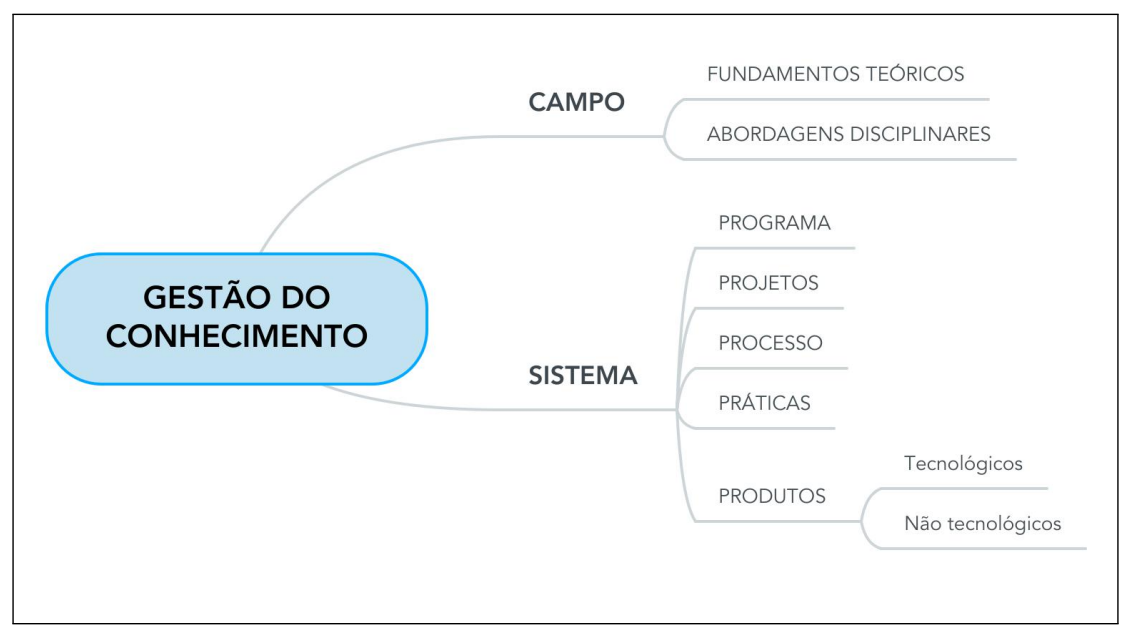

Figura 2. Categorias de Gestão do Conhecimento.

Fonte: Elaboração própria a partir da ferramenta Mindmeister (2020).

Em busca de uma melhor visualização das categorias e suas relações hierárquicas, chegou-se à sistematização representada na Figura 2.

É importante reforçar que as categorias de GC foram definidas a partir da análise da abordagem principal dos autores em suas respectivas obras, identificando palavras-chave que representassem explicitamente (considerando o uso da palavra no texto) ou implicitamente (considerando a referência indireta à palavra a partir do texto) tais abordagens. Segue a descrição de cada uma das categorias e suas subcategorias correspondentes:

\section{Campo}

Com base em Bourdieu (2004), o Campo Científico é um grupo de disciplinas estruturado em torno de objetivos e práticas específicas e relativamente autônomas com relação a outras disciplinas, integrando uma diversidade de agentes e instituições produtoras ou difusoras da ciência no interior de cada uma delas. Nesse sentido, o Campo da Gestão do Conhecimento pode ser abordado numa perspectiva teórica e prática:

- Fundamentos teóricos: A GC se caracteriza como um campo multidisciplinar, ou seja, composto pela justaposição de duas ou mais disciplinas, com foco na proximidade, em que cada disciplina contribui dentro da sua área de conhecimento (Holland, 2008). Logo, a Gestão do Conhecimento integra teorias oriundas de diversas outras disciplinas.

- Abordagens disciplinares: Assim como pode ser abordada numa perspectiva teórica, fundamentada em teorias oriundas de diversas áreas científicas, a GC também pode ser estudada como um campo aplicado, com práticas, metodologias, conceitos e abordagens associadas às diferentes disciplinas (Holland, 2008).

\section{Sistema}

A ISO 30401 (2018) define Sistema de Gestão do Conhecimento como "parte de um Sistema de Gestão no que diz respeito ao conhecimento" (ISO, 2018, p. 4), sendo o principal elemento a ser promovido. Sistema de Gestão, por sua vez, é identificado como um conjunto de elementos interdependentes que operam em um ambiente ou contexto para estabelecer políticas, objetivos e processos para alcançar esses objetivos.

- Programa: Segundo o PMBOK (PMI, 2017, p. 8), programa é um "grupo de projetos gerenciados de uma forma coordenada, a fim de se obter benefícios que, de uma forma isolada, não se obteria". No caso de um Programa de Gestão do Conhecimento, este é capaz de possibilitar o gerenciamento de diversos projetos de GC, cada um com objetivos específicos relacionados a um objetivo geral único, sendo necessário o desenvolvimento de atividades de gestão (ex: gestão de relacionamentos, gestão de políticas, gestão da tecnologia) e a promoção de elementos facilitadores (ex: cultura organizacional, liderança) para o conhecimento organizacional, cujos elementos são representados em diferentes modelos organizacionais de gestão do conhecimento.

- Projetos: Segundo o PMBOK (PMI, 2017, p. 4)), um projeto é um "empreendimento temporário com o objetivo de criar um produto ou serviço único, que pode ser desenvolvido em qualquer nível da organização". Ainda segundo o documento, "“temporário" significa que cada projeto tem um começo e um fim bem definidos e "único" significa que o produto ou serviço produzido é de alguma forma diferente de todos os outros produtos ou serviços semelhantes" (PMI, 2017, p. 4). No caso do projeto de GC, destaca-se que os objetivos e as metas estão relacionados ao desempenho que a organização pretende alcançar e qual a lacuna de conhecimento deverá ser preenchida. 
- Processo: Considera a classificação do conhecimento em duas categorias: o conhecimento explícito (objetivo, formal e codificado) e o conhecimento tácito (subjetivo, informal e não codificado). Com base nessa classificação, refere-se aos quatro modos de conversão do conhecimento (explícito e tácito), representando a interação social entre os indivíduos como socialização, combinação, internalização e externalização, cuja representação já é bem conhecida. Refere-se, ainda, às etapas do processo de gestão do conhecimento, resumidas em aquisição/criação, armazenamento, compartilhamento e utilização do conhecimento, cujas etapas são representadas em diferentes modelos de processo em gestão do conhecimento.

- Práticas: Sinônimo de "ato ou efeito de realizar algo" (Dicionário Michaelis, 2010), o conceito de "prática" pode ser definido, quando aplicado no contexto da Gestão do Conhecimento, como uma ação capaz de concretizar aquilo que a GC se propõe a fazer na sua teoria, ou seja, o seupromover o processo de gestão do conhecimento para aumentar a eficiênciaefetividade, melhorar a qualidade e promover a inovação a partir de um método integrado.

- Produtos: Em um sistema de gestão do conhecimento, os produtos de GC (tecnológicos ou não tecnológicos) devem ser entendidos como aqueles que podem ser aplicados para promover facilitar a promoção de práticas em gestão do conhecimento, a partir do seu processo, ou podem ser resultados dessas ações. Um exemplo do primeiro caso são as páginas amarelas, que indicam pessoas com conhecimentos específicos em determinada área, setor ou assunto, e que serve de suporte para o compartilhamento do conhecimento a partir de comunidades de prática, por exemplo. O segundo caso ocorre quando as comunidades de prática podem gerar como resultado as lições aprendidas relacionadas a uma problemática.

\section{CONSIDERAÇÕES FINAIS}

A partir da revisão sistemática de literatura, foi possível delimitar o domínio da gestão do conhecimento em duas grandes categorias, sendo a primeira dividida em duas subcategorias e a segunda em cinco. A categoria Campo está relacionada aos fundamentos teóricos e abordagens disciplinares, ou seja, caracteriza a GC como um campo multidisciplinar, composto por um conjunto de fundamentos teóricos (teorias) e abordagens disciplinares (métodos, práticas e conceitos) emprestadas de outras áreas/disciplinas. Já a categoria Sistema integra um conjunto de elementos aplicados em um contexto social/organizacional, seja Programa, Projetos, Processo, Práticas ou Produtos (tecnológicos e não tecnológicos).

O resultado desta pesquisa em desenvolvimento subsidiará a composição da Taxonomia-de-base-em-conceito para a Gestão do Conhecimento. Como etapas seguintes deste trabalho, pretende-se categorizar os conceitos com base nas suas diferenças e similaridades. Além disso, os conceitos de cada categoria passarão por um processo de sistematização em cadeias hierárquicas, cumprindo assim o objetivo geral da pesquisa que é a criação de uma taxonomia para a Gestão do Conhecimento. Tal resultado poderá minimizar a confusão conceitual existente entre especialistas, promovendo melhor comunicação e também o crescimento dessa especialidade. 


\section{REFERÊNCIAS}

Bourdieu, P. (2004). Usos sociais da ciência: por uma sociologia clínica do campo científico. São Paulo: Unesp.

Campos, M. d. A., \& Gomes, H. E. (2008). Taxonomia e classificação: o princípio de categorização. DataGramaZero: revista de Ciência da Informação, 9(13), 5. Recuperado de http://www.enancib.ppgci.ufba.br/artigos/GT2--101.pdf

Campos, M. d. A., Gomes, H. E., \& Oliveira, L. d. L. (2013). As categorias de ranganathan na organização dos conteúdos de um portal científico. DataGramaZero: Revista de Informação, Rio de Janeiro, 14, 3. Recuperado de http:// www.brapci.inf.br/index.php/article/download/53312

Dahlberg. (1981). Conceptual definitions for interconcept. International Classification, 8(1), 16-22. Recuperado de https://www.ergon-verlag.de/isko_ko/downloads/ ic_8_1981_1_d.pdf

Dicionário Michaelis. (2010). Dicionário Brasileiro da Língua Portuguesa. Recuperado de https://michaelis.uol.com.br/moderno-portugues/busca/ portugues-brasileiro/pr\{á\}tica/

Duarte, E. N., Satur, R. V., Lira, S. d. L., Silva, N. B. X., \& Lima, E. S. d. (2015). Práxis de gestão do conhecimento no ambiente das organizações no escopo da Ciência da Informação. In Xvi encontro nacional de pesquisa em ciência da informação (xvi enancib). Recuperado de http://www.ufpb.br/evento/index.php/ enancib2015/enancib2015/paper/viewFile/2728/1078

Galvão, T. F., \& Pereira, M. G. (2014). Revisões sistemáticas da literatura: passos para sua elaboração. Epidemiologia e Serviços de Saúde, 23, 183-184. Recuperado de http://www.scielo.br/pdf/ress/v23n1/2237-9622-ress-23 -01-00183.pdf

Garza-Reyes, J. A. (2015). Lean and green-a systematic review of the state of the art literature. Journal of Cleaner Production, 102, 18-29. Recuperado de http://dx.doi.org/ 10.1016/j.jclepro.2015.04.064

Gheno, T. C. (2017). Análise de domínio: um estudo das publicações cientificas brasileiras (Dissertação de mestrado, Universidade Federal de Santa Catarina, Brasil). Recuperado de https://repositorio.ufsc.br/xmlui/bitstream/handle/ $123456789 / 179000 / 348756$.pdf sequence $=1 \&$ is Allowed $=y$

Guimarães, J. A. C. (2014). Análise de domínio como perspectiva metodológica em organização da informação. Ciência da Informação, 43(1), 13-21.

Hjørland, B. (2002). Domain analysis in information science. Journal of documentation, 58(4), 422-462. doi: https://doi.org/10.1108/00220410210431136

Holland, G. A. (2008). Information science: an interdisciplinary effort? Journal of Documentation, 64 (1), 7-23. doi: https://doi.org/10.1108/00220410810844132

Lopes, A. L. M., \& Fracolli, L. A. (2008). Revisão sistemática de literatura e metassíntese qualitativa: considerações sobre sua aplicação na pesquisa em enfermagem. Texto $\&$ Contexto-Enfermagem, 17(4), 771-778. doi: http://dx.doi.org/10.1590/S0104-07072008000400020

Organização Internacional de Normalização (ISO). (2018). ISO 30401: Sistema de Gestão do Conhecimento. Varnier: ISO. Recuperado de https://www.borhanjooyan.com/DL/ ISO-30401-2018.pdf

Project Management Institute (PMI). (2017). Guia
PMBOK@: Um Guia para o conjunto de Conhecimentos em Gerenciamento de Projetos. Pennsylvania: PMI.

Ranganathan, S. R. (1937). Prolegomena to library classification. Bombay: Asia Publishing House.

Sampaio, R. F., \& Mancini, M. C. (2007). Estudos de revisão sistemática: um guia para síntese criteriosa da evidência científica. Brazilian Journal of Physical Therapy, 11(1), 83-89. Recuperado de http://dx.doi.org/10.1590/S1413 $-35552007000100013$

Terra, J. C., Schouerl, R., Vogel, M. J., \& Franco, C. (2009). Taxonomia: elemento fundamental para a gestão do conhecimento. Recuperado de https://pt.slideshare.net/jcterra/taxonomia-elemento -fundamental-para-a-gestao-do-conhecimento
Como citar este artigo (APA):

Silva, N. B. X. \& Sales, L. F. \& Santos, J. D. F. (2020). Estudo de categorias para sistematização de conceitos em gestão do conhecimento. AtoZ: novas práticas em informação e conhecimento, 9(1), 32 - 41. Recuperado de: http://dx.doi.org/10.5380/atoz.v9i1.74363 